\title{
Onset but not offset of irrelevant motion disrupts inhibition of return
}

\author{
RICHARD A. ABRAMS and SHAWN E. CHRIST \\ Washington University, St. Louis, Missouri
}

\begin{abstract}
In seven experiments, subjects were slower to detect targets in cued static objects than in uncued static objects, revealing inhibition of return (IOR). This occurred regardless of the presence or absence of continuous motion of other, task-irrelevant objects in the display. However, if the motion of the irrelevant objects began during the interval between cue and target, the amount of IOR was considerably reduced. Offset of motion during the cue-target interval had no effect. Implications for IOR, object perception, and attentional capture are discussed.
\end{abstract}

Inhibition of return (IOR) refers to a relative slowness to respond to stimuli at recently attended locations (Posner \& Cohen, 1984). IOR is thought to permit efficient visual searches in complex environments because it biases a search toward fresh sources of input. Consistent with this view are findings that indicate that IOR operates in an object-based reference frame. For example, Tipper, Driver, and Weaver (1991) presented two objects on an imaginary circle centered at fixation. The objects both moved partway around the circle, during which time one of them was cued by flickering. Subjects were slower to detect a subsequent target presented in the cued object (in comparison with the uncued one) even though the object had since moved to a new location. Thus, IOR appears to include an object-based component that permits inhibition of an attended object even after it moves. Subjects are also slower to detect stimuli at the original location of the cue even after the cued object has left, revealing an additional location-based component to the inhibition (Tipper et al., 1991). Several others have provided evidence of both the object- and location-based components of IOR (e.g., Abrams \& Dobkin, 1994; Abrams \& Pratt, 2000; Jordan \& Tipper, 1998; McCrae \& Abrams, 2001; Tipper, Weaver, Jerreat, \& Burak, 1994), although there are also some special situations in which the object-based component has not been observed (Abrams \& Dobkin, 1994; Abrams \& Pratt, 2000).

Despite the ecological arguments made in support of IOR, most of what is known about it comes from paradigms in which participants viewed static stimuli or from paradigms such as the one just described, in which the few relevant objects in the display moved a short distance. Such situations are artificial and not typical of real-world scenes, in which many different kinds of motion are pos-

The work reported here was supported by Grant BCS-0079594 from the National Science Foundation. Correspondence concerning this article may be sent to R. A. Abrams, Department of Psychology, Washington University, St. Louis, MO 63130 (e-mail: rabrams@wustl.edu). sible and in which motion through the environment may cause images of many objects to move across the retina. To examine IOR in dynamic scenes, we recently studied what happens when a cued object moves away from its initial location but then returns to it (Christ, McCrae, \& Abrams, 2002). Quite surprisingly, we found that the IOR was almost completely eliminated even though both objectbased and location-based components of inhibition might be expected to be operating when a cued object returns to its initial location. It is significant that our results showed that dynamic scenes may reveal properties of IOR that differ from those present in static scenes.

In the present research, we sought to extend our earlier investigation of IOR in dynamic scenes by examining situations in which the motion involves objects that are not directly relevant to the subject's task. Such a situation may be typical of many real-world searches in which the object of primary interest may be stationary or, if moving and fixated, may be the least likely to move relative to the retina. Under conditions such as these, little is known about the role that IOR might play in search. In the experiments that follow, we examine IOR under conditions in which irrelevant objects in the display undergo one of several different types of motion or do not move at all. The results reveal that some types of motion are not disruptive at all, whereas other types of motion can dramatically affect the magnitude of IOR.

The present study also provides an opportunity to examine the possibility that some types of motion have a qualitatively different impact on attention than do others (Franconeri \& Simons, 2003; but see Abrams \& Christ, 2005). In particular, we recently reported that the onset of motion attracts attention during visual search, although motion offset or continuous motion does not (Abrams \& Christ, 2003). If motion onset has a similar effect during cuing in an IOR paradigm, then it might be expected to diminish the amount of IOR observed. This is because each event that attracts attention might serve as an attentional cue, and successive cues have been shown to reduce the magnitude of IOR at the initially cued location (Abrams \& 
Pratt, 1996; Knapp \& Abrams, 2001; Snyder \& Kingstone, $2000,2001)$. Such a result would extend our knowledge of the effects of motion onset and would further serve to integrate research on visual search and on IOR.

\section{EXPERIMENT 1}

In the first experiment, we asked whether the mere presence of motion in a scene would be sufficient to reduce IOR. Tipper et al. (1994) reported a reduction in IOR for static objects when moving objects were present in a scene. However, in their experiment both moving and static objects were potentially relevant to the task and thus were presumably being attended. In the present experiment, we asked a simpler question: If objects that are clearly not relevant to the task are set in motion, will their motion affect IOR? Disruption of IOR under task-irrelevant motion is predicted by the explanations that have been offered for some of the earlier results. For example, Tipper et al. (1994) suggested that the ability to track moving objects would require the activity of cortical motion-sensitive mechanisms in addition to the midbrain mechanisms typically thought to underlie IOR. The activity of the cortical mechanisms was thought to engage an object-processing system that could suppress the inhibition that would have been present if all elements in the scene had been stationary. Christ et al. (2002) offered a similar explanation for the effects of motion in their study. If these explanations are correct, then it should be the case that any motion in a scene - even that of irrelevant objects - could potentially reduce the magnitude of IOR to static objects. To exam- ine this possibility, we conducted an experiment that was very similar to a typical experiment in IOR, but with some extraneous moving objects that were irrelevant to the task added to the display.

\section{Method}

Subjects. Each of 12 experimentally naive undergraduates from the Washington University community served in one 45-min session in exchange for course credit.

Apparatus and Procedure. Subjects were seated in front of a computer monitor in a dimly lit room. Their heads were steadied with a chinrest. Two types of trials were studied: dynamic and static. The timing and sequence of events was very similar to that which we used in the Christ et al. (2002) study. The events on a dynamic trial are illustrated in Figure 1. Each trial began with a fixation display that contained a central dot and four $1^{\circ}$ boxes. The boxes appeared at the corners of an imaginary $14^{\circ}$ square centered on the fixation point. Two of the boxes were unfilled and two were gray, with boxes of the same fill style located at diagonally opposite corners of the configuration. Cues and targets were presented only in the unfilled boxes, and the subjects were apprised of this fact. The gray boxes were irrelevant to the task and could be safely ignored throughout the entire session.

The initial display was presented for $800 \mathrm{msec}$, after which the central dot was brightened and enlarged slightly for $100 \mathrm{msec}$ before returning to its initial state. Following a 300 -msec delay, one of the unfilled boxes was cued by being brightened and filled in for $50 \mathrm{msec}$. This was followed by a $117-\mathrm{msec}$ delay. On dynamic trials, the gray boxes then each moved smoothly along a circular pathway, with the motion ending with the two boxes in their original locations. The imaginary circle demarcating the path that each box traversed was approximately $4.5^{\circ}$ in diameter and was centered on the corresponding corner of an imaginary $20^{\circ}$ square centered on the fixation point. The direction of motion of each of the boxes was such that the box located to the left of fixation (whether in the top

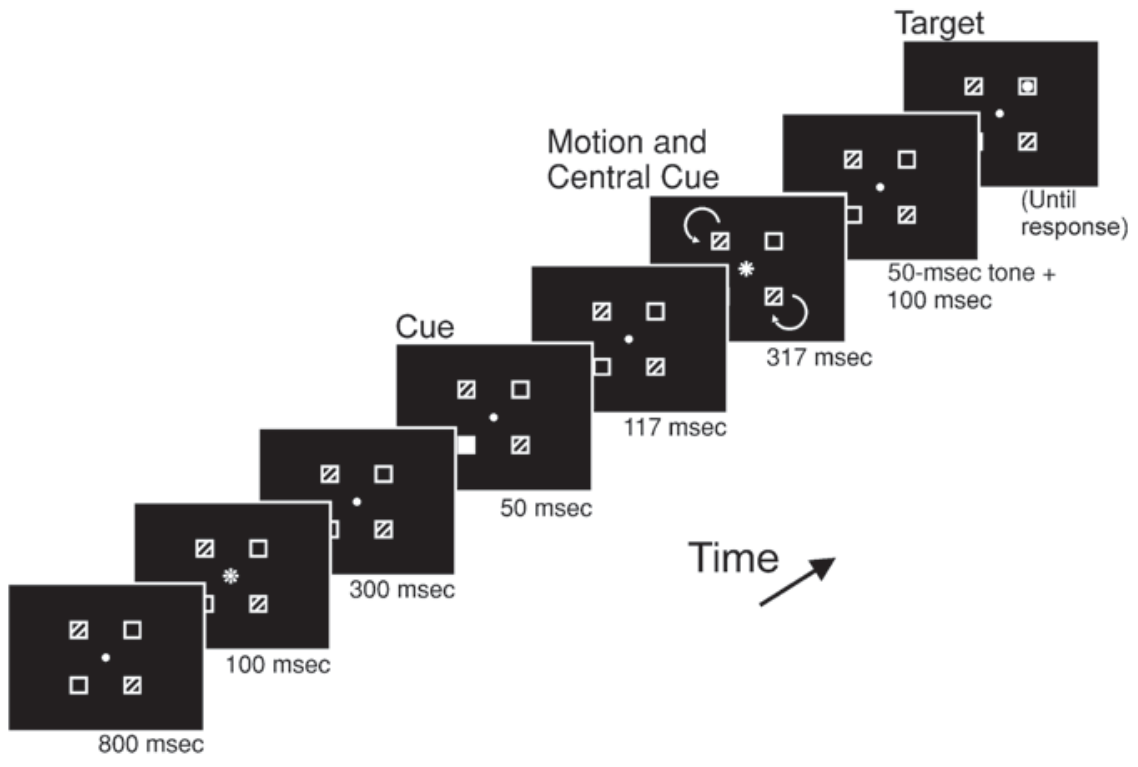

Figure 1. Sequence of events on a trial in the dynamic condition of Experiment 1. Stripes indicate boxes that were gray during the experiment. The gray boxes could never contain cues or targets and could in theory be ignored. Arrows in the sixth frame indicate the direction of motion of the boxes but were not visible in the display. In that frame, the central cue, which consisted of a 50-msec brightening of the central circle, was presented $133 \mathrm{msec}$ after the onset of motion. 
left or bottom left location) always moved in a counterclockwise direction, and the box located to the right of fixation moved clockwise around its circular path. The motion was accomplished by displaying boxes at each of 20 equally spaced positions along the motion path, each for $16.7 \mathrm{msec}$ (total motion time for the 19 displacements was $316.7 \mathrm{msec}$ ). One hundred thirty-three milliseconds after motion began, the central dot was brightened and enlarged slightly for $50 \mathrm{msec}$. After motion had ceased, a 50-msec, 400-Hz warning beep was sounded, followed by a 100-msec delay. On target-present trials, a $0.5^{\circ}$ circular target was then presented in one of the two unfilled boxes. The resulting stimulus onset asynchrony was $633 \mathrm{msec}$, which is very similar to that used in other experiments in IOR in both static and moving paradigms (e.g., Tipper et al., 1994; Weaver, Lupiáñez, $\&$ Watson, 1998). The subjects were instructed to press the space bar on the keyboard as quickly as possible when the target appeared and to refrain from responding on catch trials (during which no target was presented). Presentation on static trials was identical to that on dynamic trials, with one exception: Instead of moving, the gray boxes (as well as the unfilled ones) remained fixed at their original locations throughout the trial.

If a subject responded on a catch trial or responded less than $100 \mathrm{msec}$ after target onset (an anticipatory response), a brief tone was presented, followed by the message TOO EARLY. Conversely, if a subject failed to respond within $1,200 \mathrm{msec}$ on a target trial, a tone and the message Too SLOW. were presented. After each block, each subject was informed of his or her mean reaction time (RT) and number of errors.

Design. Following 40 practice trials, the subjects served in 360 experimental trials, 180 of which were in the dynamic condition and 180 of which were in the static condition. Twenty percent of the trials in each condition were catch trials in which no target was presented. Trial presentation was balanced so that cue and target were equally likely to appear in the same box (cued trials) and in different boxes (uncued trials), and so that each was equally likely to appear in either of the two boxes. Furthermore, the unfilled boxes were equally likely to appear in the bottom right and top left corners of the display, and in the bottom left and top right corners of the display. The trial types were randomly mixed. At intervals of 40 trials, the subjects were given the opportunity to take a break.

\section{Results}

Mean RTs on static and dynamic trials are shown in Figure 2. As can be seen in the figure, we obtained the typical IOR effect, with subjects slower to respond on cued trials than on uncued trials $[F(1,11)=25.6, p<.001]$. Static and dynamic trials did not differ overall $[F(1,11)<1]$, but there was an interaction between cuing and motion condition $[F(1,11)=14.2, p<.005]$ : Effects of cuing were smaller, revealing less IOR, in the dynamic condition $(18.2 \mathrm{msec})$ in comparison with the static condition $(44.1 \mathrm{msec}) .{ }^{1}$ Error rates are shown in Table 1, where it can be seen that the subjects were somewhat more error prone on static trials than on dynamic trials [considering only the target trials, $F(1,11)=7.45, p<.05]$, but the percentages of error did not differ across cuing conditions.

\section{Discussion}

In the present experiment, irrelevant gray boxes in the periphery of the display moved on dynamic trials. The subjects knew that the gray boxes would never contain any information related to their task. Nevertheless, the motion dramatically reduced the IOR that was observed when responses to targets presented in the task-relevant, static ob-

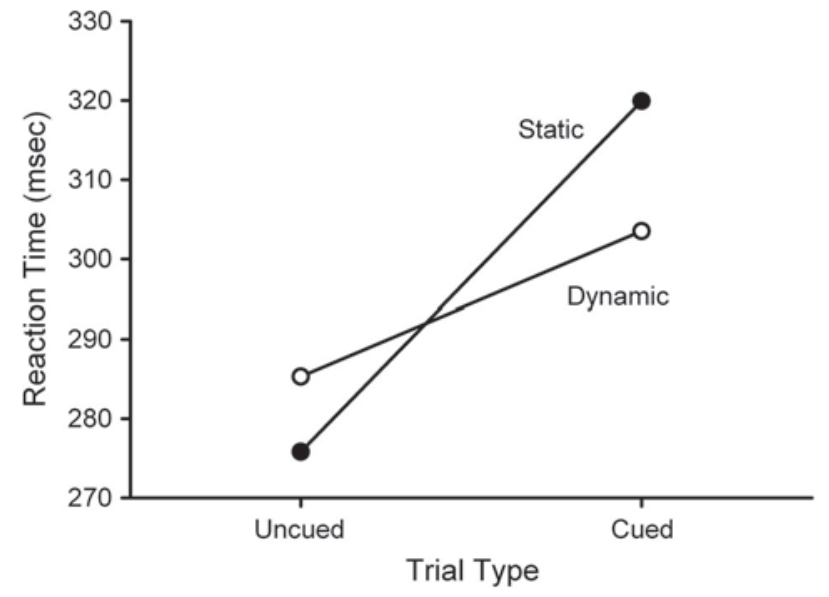

Figure 2. Mean reaction times from Experiment 1. In this and subsequent experiments, the objects that moved were irrelevant to the subject's task and could theoretically be ignored. In the dynamic condition, the irrelevant objects started and stopped moving between the presentation of cue and that of target. As a result, both an onset and an offset of motion occurred during the interval between the cue and the target. The objects did not move on the static trials.

jects were being measured. As was suggested earlier, it is possible that cortical motion-detecting mechanisms were engaged by the motion, perhaps activating an object-based reference frame (Tipper et al., 1994), and that said system suppressed the inhibition that might have otherwise been present. An alternative possibility is that some aspect of the motion attracted the subjects' attention, weakening the effect of the earlier cue (Knapp \& Abrams, 2001; Snyder $\&$ Kingstone, 2001). The remaining experiments were designed to uncover what it is about the motion that disrupts the inhibitory processes.

\section{EXPERIMENT 2}

On the dynamic trials in Experiment 1, not only did the irrelevant gray boxes move between presentation of the cue and presentation of the target, but they also started moving and stopped moving during that interval. Given the perceptual system's sensitivity to change, it seems possible that the onset and/or offset of the motion may have been as disruptive as the motion itself. To test this possibility, in the present experiment we set the irrelevant objects in motion before the cue was presented, and the objects remained moving until after the target had been presented. In this way, in the interval between cue and target, exactly the same motion occurred here as in Experiment 1 . However, in the present experiment, because the irrelevant objects were continuously moving, they neither started nor stopped moving during that interval.

\section{Method}

Subjects. Each of 12 students drawn from the same population as those in Experiment 1 served in one 45-min session. None had served previously. 
Table 1

Percent Error Rates From Each Experiment of the Present Study

\begin{tabular}{|c|c|c|c|c|c|c|c|c|}
\hline \multirow[b]{3}{*}{ Experiment } & \multirow[b]{3}{*}{ Motion Condition } & \multirow[b]{3}{*}{ Overall } & \multicolumn{6}{|c|}{ Condition } \\
\hline & & & \multicolumn{3}{|c|}{ Static } & \multicolumn{3}{|c|}{ Motion } \\
\hline & & & Catch & Cued & Uncued & Catch & Cued & Uncued \\
\hline 1 & Dynamic* & 4.4 & 13.2 & 2.7 & 2.9 & 11.1 & 1.6 & 1.5 \\
\hline 2 & Continuous motion & 4.1 & 3.5 & 4.4 & 4.3 & 5.6 & 4.1 & 3.2 \\
\hline 3 & Early motion onset & 3.1 & 12.3 & 1.5 & 1.0 & 10.6 & 0.9 & 0.7 \\
\hline 4 & Late motion offset & 2.5 & 2.6 & 2.3 & 1.7 & 4.0 & 2.4 & 2.3 \\
\hline 5 & Early motion offset & 3.5 & 8.7 & 2.0 & 1.5 & 9.4 & 2.5 & 2.5 \\
\hline 6 & Late motion onset & 3.3 & 3.1 & 2.7 & 1.9 & 6.9 & 3.6 & 3.2 \\
\hline 7 & Early motion onset & 2.8 & 6.2 & 1.7 & 1.6 & 8.2 & 2.1 & 0.8 \\
\hline
\end{tabular}

*In Experiment 1, with only the trials with targets accounted for, more errors were made in the static condition than in the dynamic condition $[F(1,11)=7.45, p<.05]$. No other differences in error rates were observed.

\begin{abstract}
Apparatus, Procedure, and Design. This experiment was very similar to Experiment 1, with the differences noted here. The static condition was identical to that of Experiment 1. In the continuous motion condition, the two gray boxes moved as they had in Experiment 1 except that the motion began at the beginning of the trial. The cue was presented (always in an unfilled box) 1,200 msec after motion onset. As in Experiment 1, the target appeared in either the cued or the uncued (unfilled) box $633 \mathrm{msec}$ after the cue onset. Motion of the gray (task-irrelevant) boxes continued until after the subject responded.
\end{abstract}

\section{Results and Discussion}

Mean RTs for static and continuous motion conditions are shown in Figure 3. The subjects were slower to respond to targets in the cued condition than to those in the uncued condition, revealing a typical IOR effect $[F(1,11)=24.5, p<.001]$. There was no effect of motion condition $[F(1,11)=4.2, p>.05]$ and no interaction between cuing and motion condition $[F(1,11)<1]$. Thus, in the present experiment continuous motion of irrelevant objects had no effect on the amount of IOR affecting the relevant objects. (IOR magnitude was $42.8 \mathrm{msec}$ in the static condition and $39.7 \mathrm{msec}$ in the continuous motion condition.) This is surprising because the motion of the irrelevant objects was identical to that studied in Experiment 1 , in which we found a substantial reduction in IOR caused by the motion. Clearly, we can now rule out the explanation offered earlier. According to that explanation, the presence of motion engaged some mechanism that worked to suppress the inhibition that would have been expected in a completely static display. Such a mechanism would also be expected to be operating in the present experiment because the same motion was present here, yet no reduction in IOR was observed. The key (and only) difference between the motion in the two experiments is that here the irrelevant objects were in continuous motion between presentation of cue and that of target, whereas in Experiment 1 the motion began and ended during the cue-target interval. We conclude that something about the onset of motion, the offset of motion, or both resulted in the observed reduction in IOR.

Error rates, shown in Table 1, did not depend on motion or cuing condition.

\section{EXPERIMENTS 3-6}

Taken together, the results of Experiments 1 and 2 suggest that the aspect of the motion of irrelevant objects that had the greatest impact on IOR was the onset of motion, the offset of motion, or both-continuous motion had no effect on IOR. In Experiments 3-6, we sought to determine whether or not the reduction in IOR could be attributed specifically to either motion onset or motion offset. To that end, we manipulated the presence or absence of motion onset and offset in the interval between cue and target. We also manipulated the relative time, during the cue-target interval, at which the motion onset or offset occurred. In each experiment, we compared static stimuli with dynamic stimuli. The condition with dynamic stimuli in each experiment contained either an onset or an offset of motion, with the onset or offset occurring either early or late in the cue-target interval. For ease of exposition,

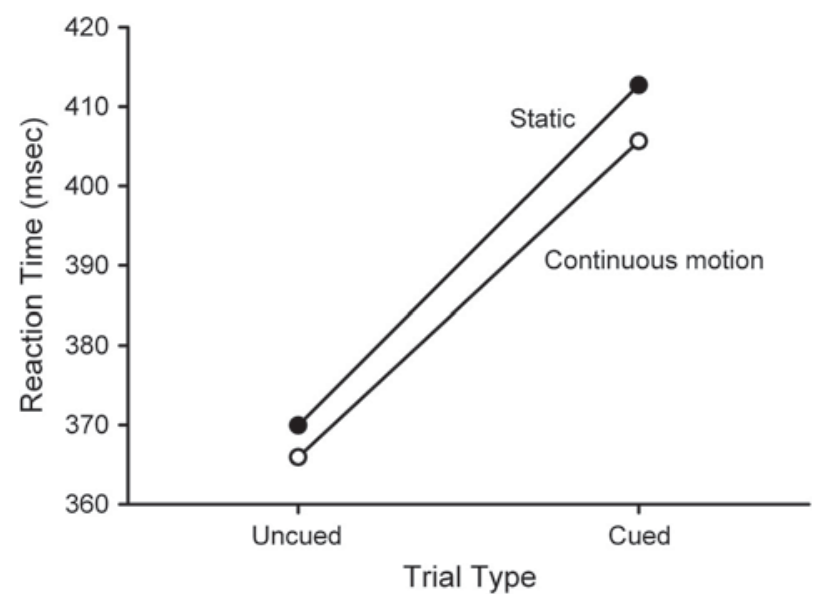

Figure 3. Mean reaction times from Experiment 2. In the continuous motion condition, the irrelevant objects were in motion continuously from before cue presentation until after the subject responded. Hence, neither an onset nor an offset of motion occurred during the cue-target interval. The objects did not move on the static trials. 
because the experiments are very similar to each other, we will describe them together.

\section{Method}

Subjects. Three groups of 16 students (one group for each of Experiments 3, 5, and 6) and one group of 17 students (Experiment 4) drawn from the same population as those who served in Experiments 1 and 2 served in the present experiment. Each subject participated in one 45-min session. None had served previously.

Procedure and Design. Each group of subjects experienced a static condition and a dynamic condition. The static condition was the same for all experiments and was identical to the static condition studied in Experiments 1 and 2. The dynamic conditions were as follows. For Experiment 3, we included an early motion onset condition, which was very similar to the dynamic condition of Experiment 1. As in Experiment 1, motion of the gray boxes commenced $167 \mathrm{msec}$ after onset of the cue. However, unlike in Experiment 1, here the motion continued until after the subject responded. Subjects in Experiment 4 experienced a late motion offset condition. In this condition, motion of the irrelevant gray squares began prior to presentation of the cue and ceased $483 \mathrm{msec}$ after cue onset (150 msec before target presentation). The time of motion offset was the same as that in the dynamic condition of Experiment 1, in which motion was found to disrupt IOR. In Experiment 5, the dynamic condition was the early motion offset condition. In that condition, motion of the irrelevant objects began prior to presentation of the cue but ceased $167 \mathrm{msec}$ after cue onset. The time of motion offset was the same as the time at which motion onset occurred in Experiment 1. The subjects in Experiment 6 were tested in the late motion onset condition, in which motion began $483 \mathrm{msec}$ after cue onset and continued until after the subject had responded. All other aspects of the design and procedure were the same as in Experiments 1 and 2.

\section{Results and Discussion}

Mean RTs for Experiments 3-6 are shown in Figure 4.

Experiment 3: Early motion onset. Mean RTs for Experiment 3 are shown in the top left panel of Figure 4. The subjects were slower on uncued trials than on cued trials, showing IOR $[F(1,15)=37.8, p<.001]$. There was also an overall effect of motion condition $[F(1,15)=7.1$, $p<.05]$, and cuing condition interacted with motion condition $[F(1,15)=6.4, p<.05]$. There was less IOR in the early motion onset condition ( $33.7 \mathrm{msec})$ in comparison with the static condition $(49.0 \mathrm{msec})$. Error rates, shown in Table 1, did not depend on motion or cuing condition. Thus, the onset of motion appears to disrupt the processes involved in IOR.

Experiment 4: Late motion offset. The data from 1 subject were excluded because of a high error rate $(>15 \%)$. All other error rates were comparable to those of the other experiments (see Table 1) and did not depend on condition. Mean RTs from each condition of Experiment 4 are shown in the bottom right panel of Figure 4 . There was an overall IOR effect $[F(1,15)=19.9, p<.001]$ and a main effect of motion condition $[F(1,15)=7.4, p<.05]$, but
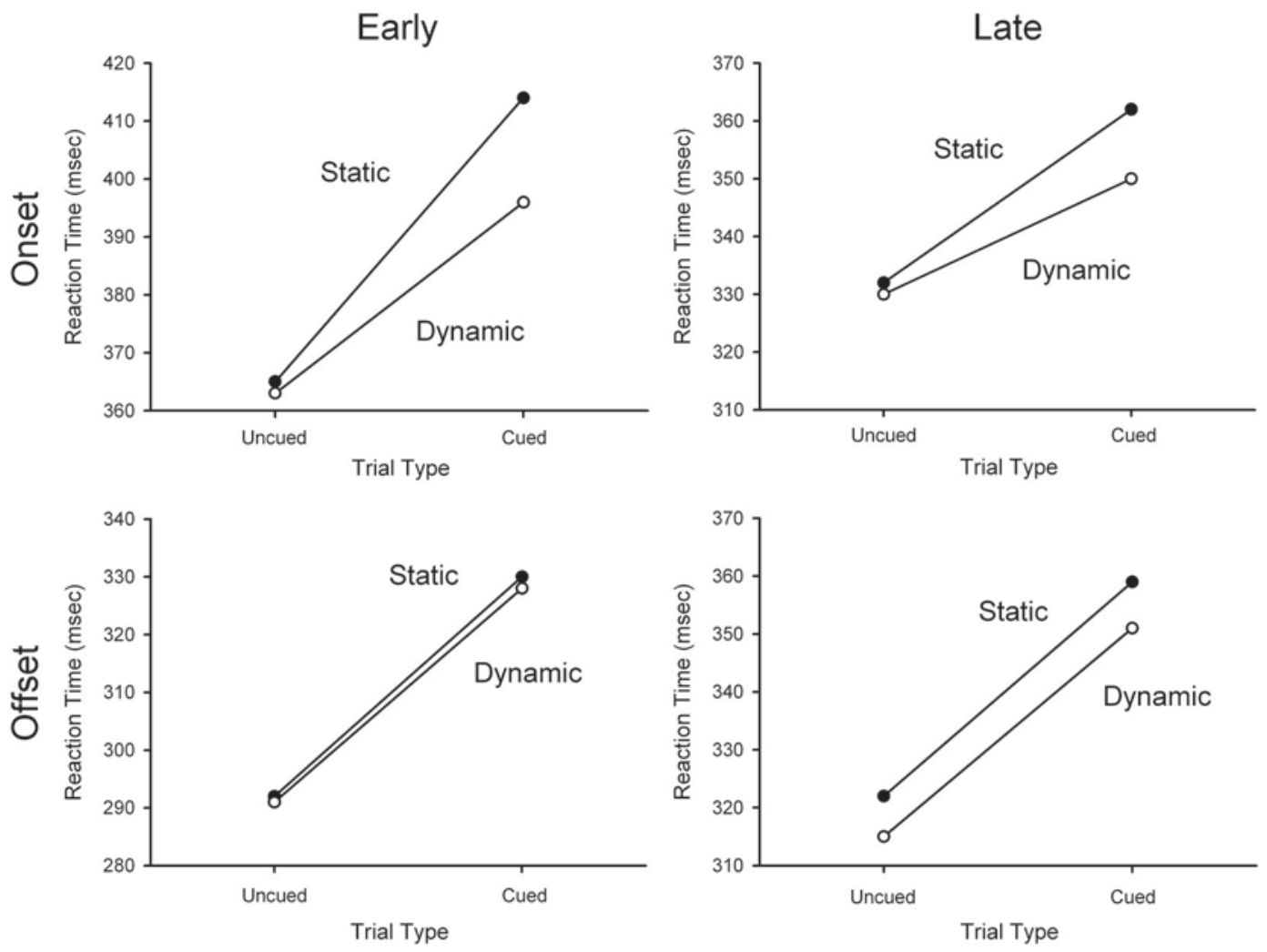

Figure 4. Mean reaction times for Experiment 3 (top left), 4 (bottom right), 5 (bottom left), and 6 (top right). The objects did not move on the static trials. On the other trials, there was either an onset or an offset of motion that occurred either early or late during the cue-target interval, as is indicated in the individual panels. 
no interaction $[F(1,15)<1]$. Thus, the offset of motion late in the cue-target interval did not affect the magnitude of IOR $(M=35.5 \mathrm{msec})$ in comparison with a static condition without any motion at all $(M=36.2 \mathrm{msec})$.

Experiment 5: Early motion offset. Mean RTs for Experiment 5 are shown in the bottom left panel of Figure 4. The subjects were slower to detect targets in previously cued locations, revealing IOR $[F(1,15)=56.0, p<$ $.001]$. However, there was no difference between the static and the early motion offset conditions, nor did the effects of the two factors interact $\left[F_{\mathrm{s}}(1,15)<1\right]$. The mean IOR magnitude was $37.5 \mathrm{msec}$ in the static condition and $36.6 \mathrm{msec}$ in the early motion offset condition.

Experiment 6: Late motion onset. Mean RTs for Experiment 6 are shown in the top right panel of Figure 4. In this experiment, we obtained an IOR effect $[F(1,15)=$ $42.2, p<.001]$. Furthermore, the amount of IOR was significantly less under late motion onset conditions than under static conditions, resulting in an interaction between the two factors $[F(1,15)=4.7, p<.05]$. The mean IOR magnitude was $29.4 \mathrm{msec}$ in the static condition and $19.7 \mathrm{msec}$ in the late motion onset condition. Motion condition did not have a main effect $[F(1,15)=2.00, p=$ .18].

Combined analysis of RTs. A mixed-model ANOVA was used to compare the RT results across Experiments 36 . The timing of the motion transient (early vs. late) and type of motion transient (motion onset vs. motion offset) served as between-subjects factors. Cuing (cued vs. uncued) and motion condition (static vs. motion transient) were entered as within-subjects factors. Most importantly, an interaction between type of motion transient, motion condition, and cuing was observed $[F(1,60)=5.84, p<$ $.05]$. In addition, the magnitude of this relationship did not appear to be further dependent on the timing of motion transient $[F(1,60)<1$, n.s.]. Main effects for motion condition $[F(1,60)=12.95, p<.001]$ and cuing $[F(1,60)=$ $129.47, p<.001]$ as well as an interaction between these two variables $[F(1,60)=7.59, p<.01]$ were also seen. No other main effect or interaction was significant $(p>$ $.05)$.

\section{Summary of Experiments 3-6}

As can be seen in the top two panels of Figure 4, when the onset of motion of the irrelevant squares occurred between presentation of cue and target, the amount of IOR observed was significantly reduced. This occurred both when the motion onset was early and when it was late in the interval. However, as is shown in the bottom two panels of Figure 4, offset of motion had no effect on the magnitude of IOR, regardless of whether it occurred early or late in the cue-target interval. Thus, we conclude that the onset but not the offset of motion disrupts IOR. One explanation for this finding is that motion onset captures attention but motion offset does not (Abrams \& Christ, 2003). If attention was attracted to the irrelevant objects, then the magnitude of IOR at the cued location, which had been attended earlier, would be expected to be reduced (Knapp \& Abrams, 2001; Snyder \& Kingstone, 2000).

\section{EXPERIMENT 7}

The results of Experiments 3-6 suggest that the presence of a motion onset in a scene, even when it occurs in irrelevant items, disrupts IOR. This occurs regardless of the timing of the motion onset relative to the cue and target presentation. We have suggested that the IOR disruption is a result of the capture of attention by the motion onset. However, an alternate explanation exists. It is possible that the motion in the display caused the subjects to make eye movements on some proportion of the trials. More importantly, the different types of motion in the different conditions might have led to different types or frequencies of eye movements. If this were the case, then the change in the magnitude of IOR that we reported may have been produced by differences in eye movements rather than by the attentional effects that we have suggested. For example, it seems possible that subjects might have looked more frequently at the irrelevant objects when they underwent a motion onset in comparison with when they experienced a motion offset. Because eye movements are sufficient to produce IOR (Machado \& Rafal, 2004; Rafal, Calabresi, Brennan, \& Sciolto, 1989), any IOR so produced might have diminished the IOR associated with the earlier cued location. More generally, if the subjects were producing different patterns of eye movements in the different motion conditions, then it is possible that eye movements contributed to the effects that we observed. In order to rule out any contribution of eye movements, we replicated Experiment 3 while monitoring eye movements.

\section{Method}

Subjects. Each of 20 students drawn from the same population as those of the previous experiments served in one 60-min session. None had served previously.

Procedure and Design. All aspects of design and procedure were identical to those of Experiment 3, with the exception that eye position was monitored throughout each trial. Eye position was recorded using an ISCAN RK426PC eye movement monitor (ISCAN, Burlington, MA). Trials during which a subject's continued fixation could not be verified were excluded from further analyses. Importantly, the number of excluded trials did not differ on the basis of cuing or motion condition $[F(1,17)<1]$ in any instance.

\section{Results and Discussion}

The data from 2 subjects were excluded because of high error rates $(>10 \%)$. Otherwise, error rates were comparable to those of the other experiments (see Table 1) and did not depend on condition. Mean RTs for static and motion onset conditions are shown in Figure 5. There was an overall IOR effect $[F(1,17)=28.8, p<.001]$ and a main effect of motion condition $[F(1,17)=21.4, p<.001]$. Most importantly, there was an interaction between these two factors $[F(1,17)=5.4, p<.05]$. In particular, the amount of IOR was significantly smaller under the motion onset condition $(27.5 \mathrm{msec})$ in comparison with the 


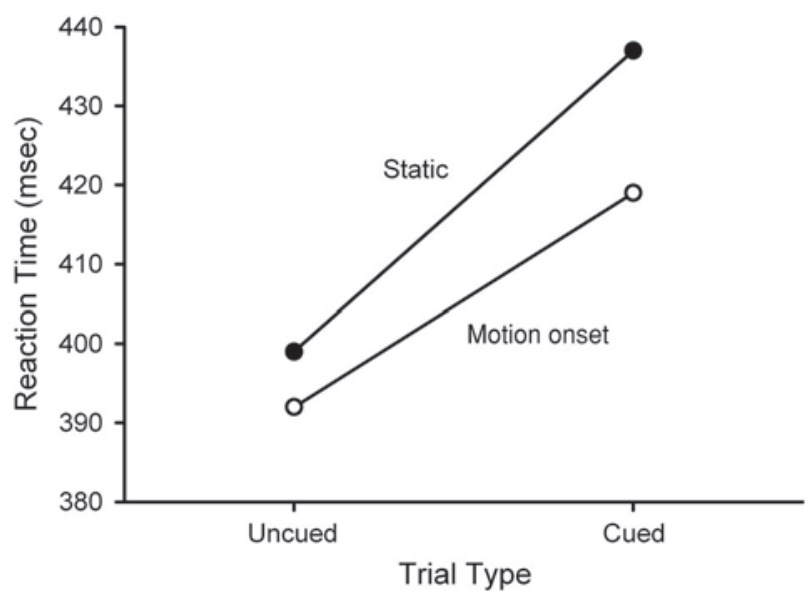

Figure 5. Mean reaction times for Experiments 7. Trials during which a subject's continued fixation could not be verified were excluded.

static condition ( $38.6 \mathrm{msec}$ ), even in the absence of eye movements. This finding rules out the possibility that the results of our previous experiments were due to the production of eye movements during the experiment.

\section{GENERAL DISCUSSION}

In the present experiments, we studied the situation in which irrelevant objects in a scene moved while subjects detected events occurring in static, attended parts of the scene. The results were straightforward: The onset of motion of the irrelevant objects was disruptive if it occurred between the time at which a relevant object was initially attended (i.e., after the cue) and the time at which a tobe-detected target was presented. The disruption took the form of a reduction in IOR, which would otherwise serve to slow responding to targets in previously attended locations. Neither the offset of motion nor continuous motion throughout the cue-target interval had a detectable effect on the magnitude of IOR. Furthermore, this disruption occurred even in the absence of eye movements.

\section{IOR in Dynamic Scenes}

Our results may help to provide new insights into how IOR operates in dynamic scenes such as those that we encounter every day. We noted earlier that the presence of motion might engage a cortical object-processing system and that such a system might suppress the inhibition that would otherwise be present in a completely static scene. This was a potential explanation for the results of our Experiment 1, and also for some of the findings of Tipper et al. (1994) and Christ et al. (2002). However, it is clear that this explanation cannot account for the absence of an effect of continuous motion in Experiment 2. This is because the motion in that experiment would have been expected to engage the cortical system in a manner similar to that caused by the motion in Experiment 1 . However, in
Experiment 2 the motion did not affect IOR at all. Thus, the mere involvement of cortical object-processing (or motion-detecting) systems is not sufficient to reduce the magnitude of IOR in static objects.

As an alternative, we suggest that IOR to static objects that are being searched in a scene is reduced when the scene contains motion that is attended. In the cases of Tipper et al. (1994) and Christ et al. (2002), the moving objects were potentially relevant to the tasks: Targets could appear in them, and thus the objects were likely to have been attended by the subjects. In the present experiments, because the moving objects were always irrelevant, the subjects presumably were not intentionally attending to them. Nevertheless, in Experiments 1, 3, 6, and 7 this motion reduced the amount of IOR. What was different about those experiments was that they contained motion onsets between the time of the initial cue and that of the subsequent target presentation. In other work, we have shown that motion onsets attract attention (whereas motion offsets and continuous motion do not; Abrams \& Christ, 2003). Thus, we conclude that the motion onsets in Experiments 1, 3, 6, and 7 captured attention, much as did the second of two cues in paradigms used to examine IOR with multiple successive cues (e.g., Abrams \& Pratt, 1996; Knapp \& Abrams, 2001; Snyder \& Kingstone, 2000, 2001). In those situations, the presence of a second cue reduced the amount of IOR that affected the first cued location, much as motion onset did in the present experiments.

\section{Relation to Object Onsets}

We found that the subjects in the present experiments were apparently unable to ignore onsets of motion despite the knowledge that the onset was irrelevant. This result is similar to the results of Theeuwes, Kramer, Hahn, and Irwin (1998), who showed that subjects had difficulty suppressing glances to new objects that appeared suddenly even though the subjects knew that those objects would never contain the target sought and were therefore not relevant to the task. In both Theeuwes et al.'s Experiment 1 and the present study, there was still uncertainty about where the target would appear, so the subjects could not restrict their attention to a single area of the display. When subjects do have complete certainty about an upcoming target location, new object onsets do not attract glances (Theeuwes et al., 1998, Experiment 2) or attention (Theeuwes, 1991; Yantis \& Jonides, 1990). Unlike object onsets, however, motion onsets do indeed appear to continue to attract attention even when subjects are certain of the target location (Abrams \& Christ, 2004).

\section{Onsets Versus Offsets}

We found a dramatic difference in the effects of motion onsets and offsets: Onsets disrupted IOR, but continuous motion and offset of motion did not. Why might motion onset be especially disruptive? One possibility is that sensitivity to motion onset may have some special adaptive significance. For example, in natural scenes, a previously 
stationary object that begins to move may represent an especially important event: That "log" in the creek may actually be an alligator-something to be wary of. But it may be very rare (as well as nonthreatening) for a moving predator to stop moving prior to an attack, and so we need not be especially sensitive to cessation of motion. If the preceding analysis is correct, then the human visual system may have evolved to be especially sensitive to motion onsets as opposed to offsets. Similar arguments have been made by Tipper and Weaver (1998), who found that IOR was reduced in displays that contained moving objects. Furthermore, responses to objects that accelerated were faster than those to objects that moved at a steady speed or decelerated. Tipper and Weaver suggested that increases in the speed of an object may attract attention because of the potential biological significance of such an event - much as we have argued for the effects of motion onset. Our hope is that future studies of dynamic scenes may further clarify the effects of motion on both facilitatory and inhibitory attentional mechanisms.

\section{REFERENCES}

Abrams, R. A., \& Christ, S. E. (2003). Motion onset captures attention. Psychological Science, 14, 427-432.

Abrams, R. A., \& Christ, S. E. (2004, May). Automatic capture of attention by the onset of motion. Poster presented at the annual meeting of the Vision Sciences Society, Sarasota, FL.

Abrams, R. A., \& Christ, S. E. (2005). The onset of receding motion captures attention: Comment on Franconeri and Simons (2003). Perception \& Psychophysics, 67, 219-223.

Aвrams, R. A., \& DobKin, R. S. (1994). Inhibition of return: Effects of attentional cuing on eye movement latencies. Journal of Experimental Psychology: Human Perception \& Performance, 20, 467-477.

Abrams, R. A., \& Pratt, J. (1996). Spatially diffuse inhibition affects multiple locations: A reply to Tipper, Weaver, and Watson (1996). Journal of Experimental Psychology: Human Perception \& Performance, 22, 1294-1298.

Abrams, R. A., \& Pratt, J. (2000). Oculocentric coding of inhibited eye movements to recently attended locations. Journal of Experimental Psychology: Human Perception \& Performance, 26, 776-788.

Christ, S. E., McCrae, C. S., \& Abrams, R. A. (2002). Inhibition of return in static and dynamic displays. Psychonomic Bulletin \& Review, 9, 80-85.

Franconeri, S. L., \& Simons, D. J. (2003). Moving and looming stimuli capture attention. Perception \& Psychophysics, 65, 999-1010.

JORDAN, H., \& TIPPER, S. P. (1998). Object-based inhibition of return in static displays. Psychonomic Bulletin \& Review, 5, 504-509.

KNAPP, W. H., III, \& ABrams, R. A. (2001, November). Automatic multilocation inhibition of return. Paper presented at the annual meeting of the Psychonomic Society, Orlando, FL.
Machado, L., \& Rafal, R. (2004). Inhibition of return generated by voluntary saccades is independent of attentional momentum. Quarterly Journal of Experimental Psychology, 57A, 789-796.

McCrae, C. S., \& Abrams, R. A. (2001). Age-related differences in object- and location-based inhibition of return of attention. Psychology \& Aging, 16, 437-449.

PosNer, M. I., \& CoHEN, Y. (1984). Components of visual attention. In H. Bouma \& D. G. Bouwhuis (Eds.), Attention and performance X: Control of language processes (pp. 531-536). Hillsdale, NJ: Erlbaum.

Rafal, R., Calabresi, P., Brennan, C., \& Sciolto, T. (1989). Saccade preparation inhibits reorienting to recently attended locations. Journal of Experimental Psychology: Human Perception \& Performance, $\mathbf{1 5}$ 673-685.

SNYDER, J. J., \& Kingstone, A. (2000). Inhibition of return and visual search: How many separate loci are inhibited? Perception \& Psychophysics, 62, 452-458.

SNYDER, J. J., \& KingSTONE, A. (2001). Inhibition of return at multiple locations in visual search: When you see it and when you don't. Quarterly Journal of Experimental Psychology, 54A, 1221-1237.

TheEUwes, J. (1991). Exogenous and endogenous control of attention: The effect of visual onsets and offsets. Perception \& Psychophysics, 49, 83-90

Theeuwes, J., Kramer, A. F., Hahn, S., \& Irwin, D. E. (1998). Our eyes do not always go where we want them to go: Capture of the eyes by new objects. Psychological Science, 9, 379-385.

TipPer, S. P., Driver, J., \& Weaver, B. (1991). Object-centered inhibition of return of visual attention. Quarterly Journal of Experimental Psychology, 43A, 289-298.

TiPPER, S. P., \& WeAVER, B. (1998). The medium of attention: Locationbased, object-centered, or scene-based? In R. Wright (Ed.), Visual attention (pp. 77-107). Oxford: Oxford University Press.

Tipper, S. P., Weaver, B., Jerreat, L. M., \& Burak, A. L. (1994). Object-based and environment-based inhibition of return of visual attention. Journal of Experimental Psychology: Human Perception \& Performance, 20, 478-499.

WeAVER, B., LuPiáÑ̃EZ, J., \& WATSON, F. L. (1998). The effects of practice on object-based, location-based, and static-display inhibition of return. Perception \& Psychophysics, 60, 993-1003.

Yantis, S., \& Jonides, J. (1990). Abrupt visual onsets and selective attention: Voluntary versus automatic allocation. Journal of Experimental Psychology: Human Perception \& Performance, 16, 121-134.

\section{NOTE}

1. We reanalyzed the results from Experiment 1 by computing median RTs instead of mean RTs. The results were nearly identical to those for means: We found a main effect of cuing $[F(1,11)=19.1, p<.005]$, an interaction between motion condition and cuing $[F(1,11)=15.83$, $p<.005]$, and no main effect of motion condition. The magnitude of IOR was $18 \mathrm{msec}$ in the dynamic condition and $44 \mathrm{msec}$ in the static condition.

(Manuscript received November 11, 2004; revision accepted for publication March 2, 2005.) 